\title{
Increased Clinical Pregnancy Outcomes; of Vitrified Blastocyst on Different Days of Embryo Development: Day 5 Compared to Day 6 Transfer
}

\author{
Mustafa Zakaria ${ }^{1}$, Marcuse F. Steven ${ }^{2 *}$, Noureddine Louanjli3 ${ }^{3}$, Wassym R. Senhaji ${ }^{4}$, \\ Abdelhafid Natiq5, Mohammed Zarqaoui6 \\ ${ }^{1}$ Department of Reproductive Biology, IRIFIV Fertility Center, IVF Laboratoy, Association for Scientific Research of the \\ IRIFIV-AISRG Group, Casablanca, Morocco \\ ${ }^{2}$ Royal Center of Obstetrics and Gynecology, Association for Scientific Research of the IRIFIV-AISRG Group, Liverpool, England \\ ${ }^{3}$ LABOMAC Laboratory, and IRIFIV Fertility Center, AFC Fertility Center, Association for Scientific Research of the \\ IRIFIV-AISRG Group, Casablanca, Morocco \\ ${ }^{4}$ Les Iris Clinc, Obstetrics and Gynecology and Association for Scientific Research of the IRIFIV-AISRG Group, \\ Casablanca, Morocco \\ ${ }^{5}$ Faculty of Medicine and Pharmacy, Mohammed V University in Rabat, Team of Genomics and Molecular Epidemiology of \\ Genetic Diseases (G2MG), Genomic Center of Human Pathologies (GENOPATH), Association for Scientific Research of the \\ IRIFIV-AISRG Group, Rabat, Morocco \\ ${ }^{6}$ IRIFIV Center Fertility, Obstetrics and Gynecology and Association for Scientific Research of the IRIFIV-AISRG Group, \\ Casablanca, Morocco \\ Email: *dr.zakaria@irifiv-aisrg.com
}

How to cite this paper: Zakaria, M., Steven, M.F., Louanjli, N., Senhaji, W.R., Natiq, A., and Zarqaoui, M. (2021) Increased Clinical Pregnancy Outcomes; of Vitrified Blastocyst on Different Days of Embryo Development: Day 5 Compared to Day 6 Transfer. Open Access Library Journal, 8: e7322.

https://doi.org/10.4236/oalib.1107322

Received: March 16, 2021

Accepted: May 4, 2021

Published: May 7, 2021

Copyright (C) 2021 by author(s) and Open Access Library Inc.

This work is licensed under the Creative Commons Attribution International License (CC BY 4.0).

http://creativecommons.org/licenses/by/4.0/

\section{(c) (i) Open Access}

\begin{abstract}
This review article demonstrates and shows the embryo transfer process in ART treatment; It also compares different embryo transfer timing and days to evaluate the pregnancy and implantation rates with day three versus day five embryo and day five versus day six embryo transfer. The review was carried out to prove that day five embryo transfer has better clinical pregnancy outcomes than day 3; thus, the embryo would spend more time developing blastocytes. Furthermore, day five embryo transfer is also preferred over day six embryo transfer since it fits the implementation window and endometrium receptivity.
\end{abstract}

\section{Subject Areas \\ Gynecology \& Obstetrics}

\section{Keywords}

Embryo Transfer, Day 5 Embryo Transfer, Day 5 vs Day 6, Embryo Culture 
and Grading, Vitrification and Warming of Blastocysts, VBT Protocol, Outcome Measures

\section{Introduction}

The in vitro fertilization method is divided into three fundamental components: induction of ovulation, oocytes fertilization, and embryos transferred back into the uterus. Ovulation induction is performed in most of the IVF cycles, while oocytes fertilization can be either achieved through in vitro fertilisation or Intracytoplasmic sperm injection (Choe, S. Archer and L. Shanks, 2020) [1]. Fertilized embryos are transferred three days after fertilization (Cleavage stage) or five days after fertilization (blastocyst stage). The blastocyst stage embryo transfer is usually associated with higher live births per cycle, fewer embryo numbers, and less multiple gestation percentage. Still, the blastocyst stage transfer disadvantages are that fewer embryos might be possible for transfer, as some embryos have been lost through the development till day five. Embryo transfer is performed under transabdominal ultrasound guidance back into the mother uterus through a catheter crossing the cervix (Choe, S. Archer and L. Shanks, 2020) [1]. This review will be discussing the embryo transfer process and techniques, comparison between different days of embryo transfer, day 5, 6 embryo transfer, and increased pregnancy outcome of the vitrified blastocyst.

\section{Embryo transfer}

The most popular technique applied in embryo transfer is transcervical transfer using transabdominal ultrasonography. Embryo transfer is usually performed on day three after oocyte fertilization or when day five of embryo development. Various catheters have made and applied for embryo transfer, where the physician is responsible for choosing the preference catheter where it does not affect the pregnancy rate (Figure 1). The embryos are transferred with $15-20 \mu \mathrm{L}$ of culture media and located in the catheter; the catheter is then inserted into the uterine cavity. The embryos ejected into the mid-cavity, about $1-2 \mathrm{~cm}$ from the fundus (Iketubosin, 2018) [2].

An embryo is usually placed into the embryo transfer catheter by a method

Centimetre depth markings on outer sheath and inner catheter

B

Figure 1. Different type of catheters used in embryo transfer. In (A) Outer Sheath a thick hollow plastic tube (catheter) with a guide catheter, stopper, and markings, it helps to blind endocervical crypts which line the cervical canal.

(B) Inner Sheath where the embryologist loads embryos into this sheath to be given to the doctor (Wallace Sure-Pro, 2021). 
called the "three-drop technique where invisible embryos and visible air bubbles managing the final position of the embryo. The air droplets are bubbled on both sides of the medium drop containing the embryo (Cenksoy and Tiras, 2014) [3]. In this method, an air bubble is first placed in the catheter. A $20 \mu \mathrm{L}$ of the medium is placed into the catheter, where the embryos are placed in the medium's smallest possible volume. Secondly, the second airdrop is then located in the catheter. Finally, an adequately medium is drawn up to make the total volume 30 $\mu \mathrm{L}$ (Figure 2). And the extra amount of fluid added to the embryo in the catheter raises the risk of embryo expulsion, while fewer fluid volumes can likewise negatively affect embryo implantation rates (Cenksoy and Tiras, 2014) [3].

After embryo loading into the catheter, it should be directly disposed into the uterine cavity to avoid the embryo's exposure to environmental conditions. The longer the embryo is exposed to the environment, the higher the risk of lower implantation and pregnancy rates. Another essential step to examine the catheter after transfer under light microscopy to identify any retained embryos. The cervix and uterus must be regularly checked as well. If untransfered embryos are detected and immediately retransferred, no harmful effects on pregnant rate should be predictable (Cenksoy and Tiras, 2014) [3].

\section{Day 5 embryo transfer}

The pregnancy percentage achieved in IVF treatment and embryo transfer has increased firmly to enhanced gamete retrieval and fertilization techniques. The improvement has been applied to embryo culture extension in vitro to the blastocyst stage as an alternative to day three embryo transfer; thus, it is considered a practical approach to establish an embryo's development potential, allowing an effective selection higher implantation rates in the following transfer (Abdelmassih et al., 2001) [4]. However, there is concern that extended in-vitro embryo culture can result in an excessive number of embryos with restricted development or with cleavage alterations related to laboratory conditions and not to deficiencies intrinsic to the embryo (Abdelmassih et al., 2001) [4]. In the (Abdelmassih et al.,

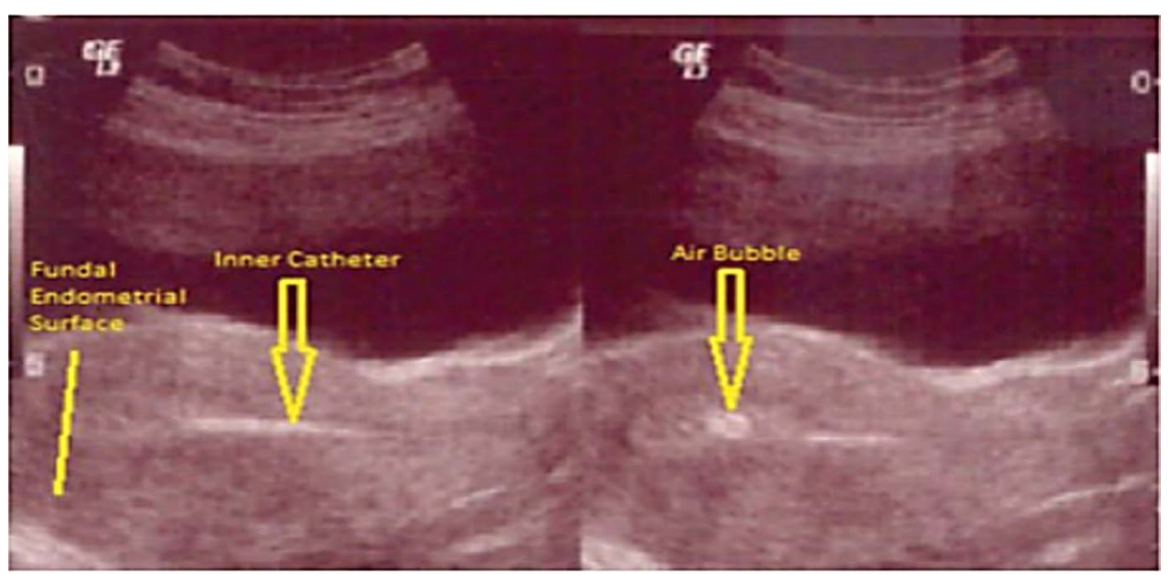

Figure 2. The location of the catheter inside the mother uterus. Where it shows the air bubble in the right images the arrow indicates the air bubble, while in the left image the arrow indicates the inner catheter (Cenksoy and Tiras, 2014). 
2001) [4] study, they attempt to perform embryo transfer on day five and day 3 ; of the 450 cycles, 296 embryo transfers were conducted on day 3 (group A); after spermatozoa microinjection, 154 embryos transfer were performed on day 5 (group B). The results indicated no difference in the development speed between group A and B of the embryos for the first $72 \mathrm{~h} .56 .3 \%$ of the embryos approached the eight-cell stage, and of those whiles, 30\% reached the blastocyst stage at $120 \mathrm{~h}$. The embryonic mortality rose through arrested development on day three (Abdelmassih et al., 2001) [4]. The average quantity of embryos transferred in group B patients was less than group A. At the same time, the pregnancy and delivery rates were higher in group B ( $45.3 \%$ and $41 \%)$ versus group A (34.8\% and 29.4\%), indicating a significantly advanced implantation rate in group B were embryos transferred at the blastocyst stage (18.5\% versus $11.5 \%$ ) (Abdelmassih et al., 2001) [4]. Moreover, the abortion percentage for both groups was almost the same, while the delivery rate was higher for day five transfers. More importantly, the multiple pregnancy rate was significantly lower in group B due almost exclusively to a drop in the triplets or more category (Abdelmassih et al., 2001) [4]. In another research, an elective single embryo transfer (SET) methodology has been implemented to match IVF goals and standers, including a healthy embryo transfer, single pregnancy and a decrease of multiple gestations (Kaye et al., 2017) [5]. It is necessary to examine all embryo selection mechanisms that support IVF treatment to achieve successful SET while decreasing pregnancy rates. Beneficial SET depends on choosing the embryo most likely to succeed. SET can be performed by extended culture to the blastocyst stage to promotes this selection, considering that genetically or developmentally impaired embryos are less likely to survive to the blastocyst stage (Kaye et al., 2017) [5]. The study conclusions support the statement of extended culture promotes SET. The results showed blastocysts cryopreserved on day six and day five. The rate of ongoing pregnancy is similar, helping the positively recommend SET. In turn, this research confirms the selection of vitrification and day six blastocyst SET to reduce the multiple gestation rate after assisted reproductive technology without an apparent decrease in pregnancy outcomes (Kaye et al., 2017) [5]. Various benefits are expected of the extended embryo transfer till the blastocyst stage: 1) In blastocyst transfer, the metabolic environment is favored of the late cleavage of human embryos, improving embryo-uterine synchrony 2) At the blastocysts stage, the uterine contractility and cervical mucus will be decreased at the embryo transfer moment. 3) Blastocyst transfers will indicate a clearer view of embryo viability and vitality, which have expressed the potential to maintain developing and differentiate in vitro and with less chromosomal abnormalities (de los Santos et al., 2014) [6].

\section{Embryo transfer day 5 vs day 6}

Embryo cryopreservation has played a crucial step in reducing the ovarian hyper-stimulation syndrome's risk and increasing ovarian stimulation cycles' efficacy in vitro fertilization treatment. Over the past years, vitrification/warming 
has been better than low-freezing/thawing regarding cryo survival rates and clinical results. It has become the favoured option for embryo cryopreservation. As a result, the use of vitrified blastocyst transfer is becoming increasingly widespread (Xu et al., 2020) [7]. Various researches have examined the IVF results of blastocyst embryo transfers on day five and day six. Nevertheless, these researches' outcomes are conflicting by suggesting that day five blastocysts result in higher pregnancy rates than day six blastocysts in fresh IVF cycles, partially as of an irregularity among the stage of embryo development and the receptivity of the recipient's endometrium (Hashimoto et al., 2013) [8]. Furthermore, another opinion suggests that those embryos transfer that reached blastocyst stage by Day 6 have the same implantation rate as their Day 5 counterparts, if they have the same morphological potential to that of Day 5 blastocyst (Sunkara et al., 2010) [9]. In Park et al., 2020, study they compare pregnancy and neonatal outcomes of day five and day six blastocysts in vitrified-warmed blastocyst transfer (VBT) cycles with propensity-score matching. The study results indicate that VBT cycles by day six blastocysts were significantly lower than those with day five blastocysts in implantation rate (IR), clinical pregnancy rate (CPR), multiple pregnancy rate (MPR) (Park et al., 2020) [10] Moreover, with day six blastocysts, the miscarriage rate was also higher in VBT cycles. Yet, no significant differences were recorded in the neonatal outcomes within the two groups. Accordingly, if there are no differences in the morphological grade between day five and day six blastocysts, then day five vitrified blastocysts is recommended to be regarded as first (Park et al., 2020) [10]. They study also conducted and found chromosomal abnormality which can illustrates the significant difference in clinical outcomes between day 5 and day 6 embryo transfer, such as implantation rate, clinical pregnancy rate. Certainly, there have been studies reporting that slower developing blastocysts have a higher aneuploidy rate. The risk of aneuploidy of day six blastocysts was $10 \%$ higher than that of day five blastocysts (Park et al., 2020) [10]. Additionally, it has been reported that irregular spindles' rate was increased, and the implantation potential was reduced after vitrification in growthretarded blastocysts in compare with normal developing blastocysts. A comprehensive analysis of the embryo by laser microscopy scanning showed a significantly higher rate of spindle irregularities in the day six vitrified embryo group than fresh developing embryos. The spindle aberrations included monopolar and abnormally shaped spindles (Hashimoto et al., 2013) [8]. In another research, they study embryo transfer timing on Day 6 blastocysts in FET cycles on clinical outcomes. They noticed that when D6 blastocysts were transferred on day five instead of day 6 , the implementation rate (IR) and clinical pregnancy rates (CPR) were significantly higher (Xu et al., 2020) [7]. As the patients' characteristics and blastocysts were similar within the tested groups, the IR and CPR difference can be considered to reflect the difference in endometrial receptivity. This can be explained as the delayed blastocyst development of D6 blastocysts ends in better synchrony with endometrial development on day 5 (Xu et al., 
2020) [7]. More interestingly, one study examined growth-retarded embryo that need six days to develop to this stage. Thus, they analysed the characteristics of vitrified-warmed human embryos that developed to day five blastocyst stage (typically developing) with the second group of embryos that required six days (growth-retarded). The results show that irregular spindles' rate was increased, and the implantation capability was reduced after vitrification in growth-retarded blastocysts compared to typically developing blastocysts. The spindle irregularities were divided into monopolar and abnormally shaped spindles. Critically, the monopolar spindles rate was significantly increased in vitrification in growthretarded blastocysts. Usually, mutations or the reserve or deficiency of several different proteins results in spindles irregularities phenotypes. On the other hand, in growth-retarded blastocysts the increase in spindle abnormalities reduced their implantation potential, still it did not change the rate of chromosomal aberrations in a bortuses (Hashimoto et al., 2013) [8]. Several studies evaluated another essential aspect: the Pre-implementation genetics screening of embryo on day five and day six and pregnancy outcome. Preimplantation genetic screening (PGS) will eventually decrease the possibility of transferring an aneuploid embryo through recognising chromosomally normal (euploid) blastocysts ere transfer (Davie et al., 2017) [11]. Transferring a euploid embryo raises the probability of a healthy, successful clinical pregnancy. Usually, blastocyst biopsy for PGS testing is performed on day 5; yet, a biopsy can be performed on day six or later, according to embryo development. The results showed that Day 5 biopsy aneuploidy percentages were similar to the overall rates. On day six, the aneuploidy rate was higher than day five embryos, both overall ( $51.3 \%$ vs $45.3 \%)$ and for each age group (Table 1). The overall aneuploidy rate for day seven biopsies (51.8\%) was similar to day 6 data (51.3\%) (Davie et al., 2017) [11].

Moreover, in Taylor et al., 2014 [12] study, they compared aneuploidy, pregnancy and live birth rates between day five and day six blastocysts. In 70 cycles, blastocysts biopsied was performed on both day five and day 6 , day five blastocysts had a higher probability of being euploid than day six blastocysts (125/229 [54.6\%]) and $(77 / 180[42.8 \%])$, respectively $(P=0.0231)$. Furthermore, euploid rates in blastocysts from patients with day 5 biopsy, day 6 biopsy, or both, were

Table 1. The aneuploidy rate for day 5 and day 6 biopsy in different age group (Davie et al., 2017) [11].

\begin{tabular}{ccc}
\hline Egg Age & \% Aneuploidy Day 5 & \% Aneuploidy Day 6 \\
\hline$<30$ & 30.8 & 36 \\
$30-34$ & 35.4 & 42.1 \\
$35-37$ & 44.1 & 50.4 \\
$38-40$ & 58.7 & 66.9 \\
$41-42$ & 75.1 & 80.1 \\
$>42$ & 83.1 & 89 \\
\hline
\end{tabular}


significantly higher in day $5(235 / 421$ [55.8\%]) compared with day $6(184 / 413$ [44.6\%]) blastocysts $(\mathrm{P}=0.0014)$ (Taylor et al., 2014) [12]. Furthermore, perspectives of ART treatment towards prolonged blastocysts development broadly change in their PGT-A. However, the use of PGT-A blastocyst-stage embryos is recognised to provide a better clinical result. Not all blastocysts represent the same pace of embryo development, and a considerable number of embryos show delay in blastulation towards day 6 or day 7 instead of day 5. In Gultomruk et al., 2019 research, the results indicated that the euploid embryos are collected from day six embryo biopsy resulted in significantly reduced clinical result compared to euploid day five embryos biopsy, although they produce good clinical pregnancies. This result implies that priority should be given today 5-biopsied embryos to obtain a better clinical outcome when there is more than one euploid embryo for a single euploid blastocyst transfer (Gultomruk et al., 2019) [13]. Several factors can cause a significant difference in clinical pregnancy rate between embryo transfer on day five and embryo transfer on day six; one of the most known ones that can be the cause of IVF is impairment of endometrial receptivity. Higher implantation rates confirm this factor in hormonal replacement treatment cycles and following ovum donation than the regular IVF cycles (Murata et al., 2005). Pinopodes have been intended as an accurate marker for endometrial receptivity through electron microscopy scanning, which allows viewing of the remarkable alteration of the exterior endometrial epithelium throughout implantation. The epithelial cells' apical layers covering the uterine cavity drop their microvilli and produce large and smooth membrane projections (Murata et al., 2005). Those pinopodes have a pinocytotic function; therefore, they are named accordingly. The pinopodes develop around one week following ovulation, and they grow and diminish within just two days. Most importantly, the development of human pinopodes is earlier in stimulation cycles (days 19 - 20) and later in hormone-controlled cycles (days 21 - 22) compared with natural processes (days $20-21$ ). These findings propose the probability that slower-growing fresh embryos may not implant due to desynchrony with the implantation window (Murata et al., 2005).

A frozen-thawed embryo's success depends on synchronization among the embryo and endometrium so that the endometrium is perfectly responsive for the embryo transfer procedure and ready for implementation. Embryo transfer timing is essential for setting the window of implantation (WOI), the time through which the endometrium becomes prepared for embryo implantation (Kim and Matevossian, 2020) [15]. The introduction of embryo transfer will open the WOI a few days later. Most recent studies suggest that the optimal WOI is small, possible not more than two days. The window is supposed to be 4 to 5 days in length, from day 20 to day 24 in an idealized 28-day cycle. It has been confirmed that several patients have dismissed WOI, which may be diagnosed through molecular markers of endometrial receptivity. For those patients, an improvement of embryo transfer initiation would better synchronize the endometrium 


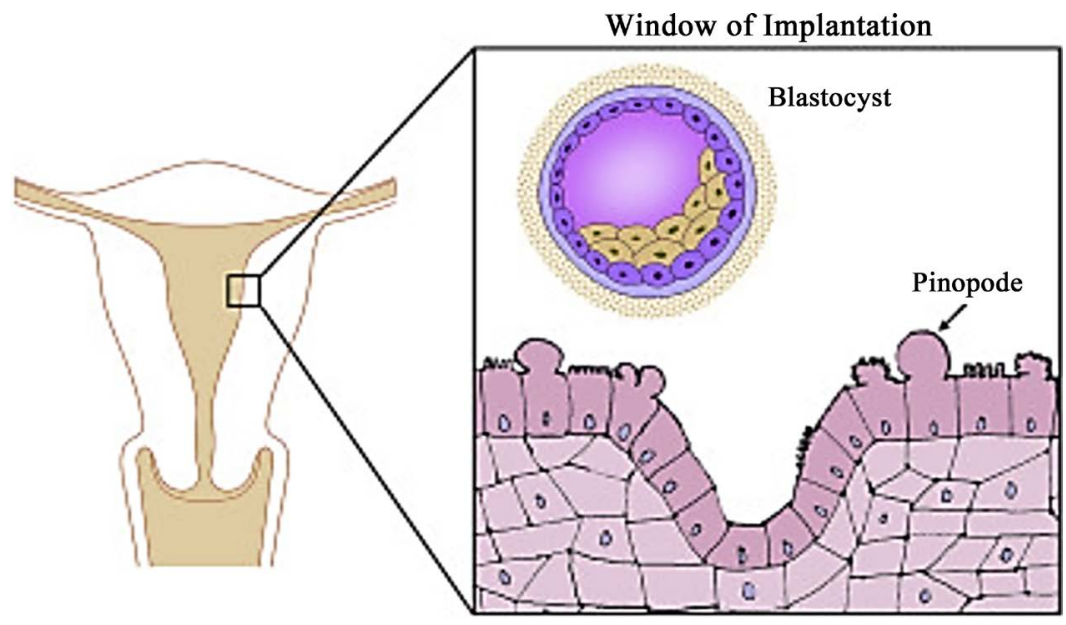

Figure 3. An illustrative graphic, which show the pinopodes location in the uterine luminal epithelium present the window of implantation (Quinn, Matson, Wetendorf and Caron, 2020) [14].

(Kim and Matevossian, 2020) [15]. Therefore, the blastocysts transfer on day six can be proposing the implantation window's without limit, pointing to decreased pregnancy rates in the fresh and vitrified cycles. Day 6 blastocysts implant at a high quality in artificially arranged endometrium, which is less advanced, leading out the importance of synchrony as a factor influencing pregnancy rates(Van Voorhis and Dokras, 2008) [16] (Figure 3).

\section{Embryo Culture and Grading}

To suppress ovulation until follicle maturity was attained, patients were treated with either a gonadotropin-releasing hormone $(\mathrm{GnRH})$ agonist or a $\mathrm{GnRH}$ antagonist. The final follicular maturation was triggered with human chorionic gonadotropin or a GnRH agonist when the mean diameter at least two leading (largest and second largest) follicles was $18 \mathrm{~mm}$. Oocytes were retrieved $36 \mathrm{~h}$ later by transvaginal ultrasound-guided needle aspiration of follicles. Conventional IVF or intracytoplasmic sperm injection (ICSI) was used for embryo fertilization. Fertilization was confirmed when two pronuclear (2PN) zygotes were observed after $16-18 \mathrm{~h}$ in ICSI and $18-20 \mathrm{~h}$ in conventional IVF. Cleavage-stage embryos were cultured in a cleavage medium (Cook, Queensland, Australia), while blastocyst-stage embryos were cultured on a blastocyst medium (Cook). Embryos were cultured in HERA cell 240 incubators (Thermo Fisher Scientific, MA, USA) in an environment with $5 \% \mathrm{O}_{2}, 6 \% \mathrm{CO}_{2}$, at $37^{\circ} \mathrm{C}$. The oil-drop culture method was applied using a four-well dish (NUNC ${ }^{\mathrm{m}}$, Thermo Fisher Scientific). At $10-\mu \mathrm{l}$ drops, the embryos were cultured individually to observe their development. Then, light paraffin oil (OVOIL, Vitrolife AB, Sweden) was dropped onto the media to prevent the medium from drying and undergoing fast $\mathrm{pH}$ change. Blastocysts were morphologically graded according to the Gardner classification [17], which takes into its expansion, inner cell mass (ICM) constitution, and trophectoderm composition prior to freezing on day 5 or 6 Blastocysts 
had good quality if all of the following criteria were met: the blastocyst expanded and filled the embryo completely (grade 3), the ICM was composed of several loosely grouped cells (grade B), and the trophectoderm contained few cells that formed loose epithelium (grade B). Blastocysts had poor quality if any of these criteria were not met.

\section{Vitrification and Warming of Blastocysts}

For blastocysts vitrification, artificial shrinkage was performed on all blastocysts, and assisted hatching was facilitated with a laser. The blastocysts were preequilibrated in hydroxyethyl piperazine-ethanesulfonic acid (HEPES) medium (Quinn's-HEPES; SAGE, in-vitro Fertilization, Inc.) supplemented with 7.5\% ethylene glycol and 7.5\% dimethyl sulfoxide (Sigma-Aldrich, St. Louis, MO, USA) for $2.5 \mathrm{~min}$ and then transferred in 15\% ethylene glycol, 15\% dimethyl sulfoxide, and 0.5-M sucrose for the final equilibration. Thereafter, the blastocysts were loaded onto an electron microscopic (EM) gold grid (EM Grid, SPI Supplies) using a fine glass pipette. The EM grids containing the blastocysts were immediately plunged into slush liquid nitrogen using VitMaster, a vitrification device (IMT Ltd., Ness Ziona, Israel). For vitrified blastocysts warming, the EM grids were sequentially transferred to culture dishes containing HEPES medium supplemented with $0.5-, 0.25-, 0.125-$, and $0.0625-\mathrm{M}$ sucrose at 2.5-min intervals, with 20\% human serum albumin (SAGE BioPharma, Bedminster, NJ). The vitrified-warmed blastocysts were washed with blastocyst medium (Cook Medical, Bloomington, IN, USA) in a $37^{\circ} \mathrm{C}$ environment with $6 \% \mathrm{CO}_{2}, 5 \% \mathrm{O}_{2}$, and $89 \% \mathrm{~N}_{2}$ and then cultured overnight.

\section{VBT Protocol}

All women underwent natural endometrial preparation. They were closely monitored for signs of dominant follicle collapse by transvaginal ultrasonography from days 10 to 12 of the menstrual cycle. Ovulation was confirmed if the follicular wall lost its clear appearance Then, luteal support was initiated using Crinone $8 \% \mathrm{w} / \mathrm{w}$ Progesterone Vaginal Gel (Merck Serono Ltd., Middlesex, UK) or vaginal progesterone Utrogestan $600 \mathrm{mg}$ (Hanhwa Pharmaceuticals, Seoul, Korea). An embryo replacement catheter (Cook) was used, and the warmed blastocysts were transferred under abdominal ultrasound guidance on day 5 after ovulation was observed. Finally, the embryo transfer catheter was checked to confirm that the embryo was no longer in the catheter.

\section{Outcome Measures}

Clinical and obstetric outcomes were as follows: implantation rate (IR), clinical pregnancy rate (CPR), multiple pregnancy rate (MPR), ectopic pregnancy rate, miscarriage rate, live birth rate (LBR). IR was calculated as the number of gestational sacs seen by ultrasonography divided by the total number of transferred blastocysts. Clinical pregnancy was defined as the presence of a fetal heartbeat 
on ultrasonogram. Miscarriage was defined as the spontaneous cessation of a clinical pregnancy before 20 gestational weeks. LBR was defined as delivery of a viable infant at $>28$ gestational weeks. Neonatal outcomes were as follows: birth weight, gestational age at delivery, and presence of malformations.

\section{Discussion}

In this study, we compared the pregnancy and neonatal outcomes of day 5 and day 6 blastocysts in VBT cycles. Our present result indicates that VBT cycles with day 6 blastocysts were significantly inferior to those with day 5 blastocysts in terms of IR, CPR, and LBR. Additionally, the miscarriage rate was higher in VBT cycles with day 6 blastocysts. However, no significant differences were found in the neonatal outcomes between the two groups. Considering these findings, if there were no differences in the morphological grade between day 5 and day 6 blastocysts, transfer of day 5 vitrified blastocysts should be considered first. However, they did not match the two groups, although the day 5 group had three times more cycles than the day 6 group. Moreover, our miscarriage rates in the day 5 and day 6 groups were similar to those in their study. Haas et al. [18] also reported a significantly lower clinical outcomes including IR and CPR Interestingly, they compared day 5 blastocysts with good-quality day 6 blastocysts ( $\geq 3 \mathrm{BB}$ ). However, they warmed day 5 blastocysts $20-24 \mathrm{~h}$ before the embryo transfer, while they warmed day 6 blastocysts $2-4 \mathrm{~h}$ prior to embryo transfer. Considering the lower clinical outcomes of day 6 blastocysts, there is still a degree of controversy in previous studies Yang et al. [19] reported that high-quality ( $\geq 3$ BB) day 6 blastocysts in VBT had similar developmental potential and pregnancy outcomes to those of high-quality day 5 blastocysts. However, they did not match day 5 and 6 groups, although the day 5 group had five times more cycles than the day 6 group. Moreover, they did not report LBR. In a meta-analysis, Sunkara et al. [9] compared the clinical outcomes of FBT with day 5 blastocysts and those with day 6 blastocysts. They included 2502 cycles from 15 controlled studies and concluded that FBT with day 6 blastocysts have similar CPR and LBR to FBT with day 5 blastocyst, if the morphological grade is the same. However, this meta-analysis had clinical heterogeneity and limited consideration of the confounders in the included studies. Although we did not perform preimplantation genetic testing for aneuploidy, chromosomal abnormality could explain the significant difference in clinical outcomes including miscarriage rate between day 5 and 6 VBT groups. Indeed, there have been studies reporting that slower developing blastocysts have higher aneuploidy rate Taylor et al. [12] reported that day 5 blastocysts had a higher chance of being euploid than day 6 blastocysts. The risk of aneuploidy of day 6 blastocysts was $10 \%$ higher than that of day 5 blastocysts. To reduce bias, they used a sibling embryo model, that is, they included patient who had biopsy on both day 5 and day 6 blastocysts in the same IVF cycles. From time-lapse culture systems, some studies revealed the close relationship between timely cell division and developmental competence 
with kinetic data in accordance with our results. Campbell et al. [20] reported that embryos having single or multiple aneuploidy had delayed initiation of blastulation compared with euploid embryos in time-lapse culture systems. An increase of spindle abnormalities in day 6 blastocysts could explain our significant results. Hashimoto et al. [8] conducted a cytoskeletal analysis of day 5 and 6 blastocysts. They found that the incidence of abnormal spindles was significantly higher in day 6 blastocysts, and IR and CPR were significantly higher in VBT of day 5 blastocysts. Interestingly, they evaluated the incidence of chromosomal abnormalities of the abortus and reported no differences between day 5 and 6 groups. They hypothesized that most blastomeres with abnormal spindles are eliminated before implantation. This hypothesis may support the safety of VBT with day 6 blastocysts, as revealed in our results. In contrast to the centers at which previous studies were conducted, our center used a natural endometrial preparation. Compared with artificial or medicated endometrial preparations, the clinical outcomes of VBT with natural endometrial preparations are not to be inferior Artificial endometrial preparations have been linked to a high miscarriage rate In addition, IVF is covered by the national health insurance system in Korea, so it is possible to perform daily ultrasonograms at low cost. For natural endometrial preparations, some clinicians use serial LH tests. However, the role of serial LH monitoring with ultrasonogram has been a subject of much debate in natural endometrial preparations and there is no clear definition of or consensus regarding LH surge Furthermore, serial LH tests are not covered by the national health insurance. Because of these reasons, our IVF center prefers to perform natural endometrial preparations without serial LH tests.

\section{Conclusion}

In conclusion, embryo transfer is one of the most crucial. Simultaneously, it is controversial since the embryo transfer can be performed on a different day following fertilization and can affect implementation rate, clinical pregnancy rate, and multiple pregnancy rate significantly. Embryo transfer on day 3 shows less clinical pregnancy rate than embryo transfer on day 5 , which indicated that embryos need to have enough time to expand, grow, and develop before transfer for better implementation. Embryos that grow to the blastocyst stage and transferred on day five following oocytes collection are most likely to implant and produce a higher clinical rate than day six embryo transfer. Several factors have been proposed, such as endometrial receptivity and implantation window.

\section{Acknowledgements}

Objective of the Association for Scientific Research of the IRIFIV-AISRG Group (IRIFIV-AISRG), Research foundation in Casablanca, Maintaining consistent and reliably high success rates are a monthly challenge for in IVF labs, the IRIFIV Fertility Center in Casablanca-Morocco Department of Reproductive Medicine and Reproductive Biology and Embryology, advocacy of interdiscipli- 
nary Department of Reproductive Medicine and Reproductive Biology and Embryology study, encompassing the areas of research, collections and publishing Articles.

\section{Conflicts of Interest}

The authors declare no conflicts of interest regarding the publication of this paper.

\section{References}

[1] Choe, J.S., Archer, J. and Shanks, A.L. (2020) In Vitro Fertilization. StatPearls, Treasure Island.

[2] Iketubosin, F. (2018) In Vitro Fertilization Embryo Transfer Processes and Pathway: A Review from Practice Perspective. Tropical Journal of Obstetrics and Gynaecology, 35, 227. https://doi.org/10.4103/TJOG.TJOG $83 \quad 18$

[3] Cenksoy, P. and Tiras, B. (2014) Practice of Embryo Transfer: Recommendations during and after. Seminars in Reproductive Medicine, 32, 291-296.

https://doi.org/10.1055/s-0034-1375181

[4] Abdelmassih, V., Balmaceda, J., Nagy, Z., Abdelmassih, S. and Abdelmassih, R. (2001) ICSI and Day 5 Embryo Transfers: Higher Implantation Rates and Lower Rate of Multiple Pregnancy with Prolonged Culture. Reproductive BioMedicine Online, 3, 216-220. https://doi.org/10.1016/S1472-6483(10)62039-1

[5] Kaye, L., Will, E., Bartolucci, A., Nulsen, J., Benadiva, C. and Engmann, L. (2017) Pregnancy Rates for Single Embryo Transfer (SET) of Day 5 and Day 6 Blastocysts after Cryopreservation by Vitrification and Slow Freeze. Journal of Assisted Reproduction and Genetics, 34, 913-919. https://doi.org/10.1007/s10815-017-0940-4

[6] de los Santos, M., Mercader, A., Galán, A., Albert, C., Romero, J. and Pellicer, A. (2014) Implantation Rates after Two, Three, or Five Days of Embryo Culture. Placenta, 14, S13-S19. https://doi.org/10.1016/S0143-4004(03)00172-3

[7] Xu, H., Qiu, S., Chen, X., Zhu, S., Sun, Y. and Zheng, B. (2020) D6 Blastocyst Transfer on Day 6 in Frozen-Thawed Cycles Should Be Avoided: A Retrospective Cohort Study. BMC Pregnancy and Childbirth, 20, Article No. 519. https://doi.org/10.1186/s12884-020-03224-Z

[8] Hashimoto, S., Amo, A., Hama, S., Ito, K., Nakaoka, Y. and Morimoto, Y. (2013) Growth Retardation in Human Blastocysts Increases the Incidence of Abnormal Spindles and Decreases Implantation Potential after Vitrification. Human Reproduction, 28, 1528-1535. https://doi.org/10.1093/humrep/det059

[9] Sunkara, S., Siozos, A., Bolton, V., Khalaf, Y., Braude, P. and El-Toukhy, T. (2010) The Influence of Delayed Blastocyst Formation on the Outcome of Frozen-Thawed Blastocyst Transfer: A Systematic Review and Meta-Analysis. Human Reproduction, 25, 1906-1915. https://doi.org/10.1093/humrep/deq143

[10] Park, D., Kim, J., Chang, E., Lee, W., Yoon, T. and Lyu, S. (2020) Obstetric, Neonatal, and Clinical Outcomes of Day 6 vs. Day 5 Vitrified-Warmed Blastocyst Transfers: Retrospective Cohort Study with Propensity Score Matching. Frontiers in Endocrinology, 11, 499. https://doi.org/10.3389/fendo.2020.00499

[11] Davie, J., Neitzel, D., Robinson, K., Zhu, M. and Faulkner, N. (2017) Aneuploidy Rates in Day 5 vs Day 6 Biopsies. Fertility and Sterility, 108, e283-e284. https://doi.org/10.1016/j.fertnstert.2017.07.840

[12] Taylor, T.H., Patrick, J.L., Gitlin, S.A., Wilson, J.M., Crain, J.L. and Griffin, D.K. (2014) Comparison of Aneuploidy, Pregnancy and Live Birth Rates between Day 5 
and Day 6 Blastocysts. Reproductive BioMedicine Online, 29, 305-310. https://doi.org/10.1016/j.rbmo.2014.06.001

[13] Gultomruk, M., Findikli, N., Boynukalin, F., Caferler, J. and Bahceci, M. (2019) Does Euploidy on Day 5 or Day 6 Matter for Clinical Outcome in Frozen Single Blastocyst Transfers? Reproductive BioMedicine Online, 38, e55-e56.

https://doi.org/10.1016/j.rbmo.2019.03.089

[14] Quinn, K., Matson, B., Wetendorf, M. and Caron, K. (2020) Pinopodes: Recent Advancements, Current Perspectives, and Future Directions. Molecular and Cellular Endocrinology, 501, Article ID: 110644. https://doi.org/10.1016/j.mce.2019.110644

[15] Kim, H. and Matevossian, K. (2020) It's All about Timing: Is the Window of Implantation Different for Day 5 and 6 Blastocysts? Fertility and Sterility, 114, 69-70. https://doi.org/10.1016/j.fertnstert.2020.04.031

[16] VanVoorhis, B. and Dokras, A. (2008) Delayed Blastocyst Transfer: Is the Window Shutting? Fertility and Sterility, 89, 31-32.

https://doi.org/10.1016/j.fertnstert.2007.01.172

[17] Gardner, D. and Lane, M. (2000) Handbook of in Vitro Fertilization. 2nd Edition, CRC Press, Boca Raton.

[18] Haas, J., Meriano, J., Laskin, C., Bentov, Y., Barzilay, E., Casper, R.F., et al. (2016) Clinical Pregnancy Rate Following Frozen Embryo Transfer Is Higher with Blastocysts Vitrified on Day 5 than on Day 6. Journal of Assisted Reproduction and Genetics, 33, 1553-1557. https://doi.org/10.1007/s10815-016-0818-x

[19] Yang, H., Yang, Q., Dai, S., Li, G., Jin, H., Yao, G., et al. (2016) Comparison of Differences in Development Potentials between Frozen-Thawed D5 and D6 Blastocysts and Their Relationship with Pregnancy Outcomes. Journal of Assisted Reproduction and Genetics, 33, 865-872. https://doi.org/10.1007/s10815-016-0712-6

[20] Campbell, A., Fishel, S., Bowman, N., Duffy, S., Sedler, M. and Hickman, C.F.L. (2013) Modelling a Risk Classification of Aneuploidy in Human Embryos Using Non-Invasive Morphokinetics. Reproductive BioMedicine Online, 26, 477-485. https://doi.org/10.1016/j.rbmo.2013.02.006

\section{Abbreviations}

$\begin{array}{ll}\text { IVF } & \text { In vitro fertilization } \\ \text { SET } & \text { single embryo transfer } \\ \text { VBT } & \text { vitrified-warmed blastocyst transfer } \\ \text { IR } & \text { implantation rate } \\ \text { CPR } & \text { clinical pregnancy rate } \\ \text { MPR } & \text { multiple pregnancy rate } \\ \text { PGS } & \text { Preimplantation genetic screening } \\ \text { WOI } & \text { window of implantation } \\ \text { MPR } & \text { multiple pregnancy rate } \\ \text { LBR } & \text { live birth rate } \\ \text { ICM } & \text { inner cell mass } \\ \text { ICSI } & \text { intracytoplasmic sperm injection } \\ \text { GnRH } & \text { gonadotropin-releasing hormone } \\ \text { EM } & \text { electron microscopic } \\ \text { FBT } & \text { frozen-thawed blastocyst transfer }\end{array}$

